\title{
ON A PROPERTY OF MONOTONE AND CONVEX FUNCTIONS
}

G. SZEKERES

We shall deal with real functions which have continuous second derivatives in some open interval $(a, b),-\infty \leqq a<b \leqq \infty$. The interval of definition of $f(x)$ is denoted by $I(f) . \phi(x)$ is called convex (from below) if $\phi^{\prime \prime}(x) \geqq 0$, concave if $\phi^{\prime \prime}(x) \leqq 0$ in $I(\phi)$.

If $\phi(x)$ is monotone increasing and convex, and $\psi_{1}(x)$ is monotone increasing and concave such that the range of $\phi(x)$ is contained in $I\left(\psi_{1}\right)$, then

$$
f(x)=\psi_{1}(\phi(x))
$$

is also monotone increasing, but usually neither convex nor concave. The question arises, under what conditions can $f(x)$ be represented in the form (1).

THEOREM 1. If $f(x)$ is strictly monotone increasing and has a continuous second derivative in $I(f)$ then it has a representation (1).

Theorem 1 states that there is a strictly increasing concave function $\psi(u)$ with continuous second derivative such that $\psi_{1}(u)=f(\psi(u))$ is concave. This is equivalent to

$$
\psi_{1}^{\prime \prime}(u)=f^{\prime \prime}(\psi(u))\left[\psi^{\prime}(u)\right]^{2}+f^{\prime}(\psi(u)) \psi^{\prime \prime}(u) \leqq 0,
$$

or if $\phi(x)$ is the inverse of $\psi(u)$, to

$$
f^{\prime \prime}(x) / f^{\prime}(x) \leqq \phi^{\prime \prime}(x) / \phi^{\prime}(x) .
$$

Let $f_{+}^{\prime \prime}(x)$ denote $f^{\prime \prime}(x)$ if $f^{\prime \prime}(x) \geqq 0$ and 0 if $f^{\prime \prime}(x)<0$. Consider the function

$$
\phi_{0}(x)=\int_{d}^{x} e^{p(y)} d y
$$

where $d$ is any fixed number in $(a, b)$ and

$$
p(y)=\int_{d}^{y}\left[f_{+}^{\prime \prime}(t) / f^{\prime}(t)\right] d t .
$$

Clearly $\phi_{0}^{\prime}(x)>0$ and

Received by the editors February 21, 1955 and, in revised form, May 26, 1955. 


$$
\phi_{0}^{\prime \prime}(x) / \phi_{0}^{\prime}(x)=f_{+}^{\prime \prime}(x) / f^{\prime}(x) \geqq f^{\prime \prime}(x) / f^{\prime}(x)
$$

so that (3) is satisfied, also

$$
\phi_{0}^{\prime \prime}(x) \geqq 0 .
$$

This proves the theorem. ${ }^{1}$

If $f(x)$ is bounded and $I(f)$ is finite, the question comes up whether $\phi(x)$ itself can be chosen to be bounded. This is answered by

THEOREM 2. If $f(x)$ is bounded, strictly increasing and has a continuous second derivative in $(a, b)$, then it can be represented in the form (1) with bounded $\phi(x)$ if and only if the integral

$$
\int_{d}^{b} e^{p(y)} d y
$$

converges, where $a<d<b$ and $p(y)$ is the function defined under (5).

We shall see presently that boundedness of $f(x)$ does not necessarily imply finiteness of (8).

To prove Theorem 2 we first note that, by $(4), \phi_{0}(x)$ is bounded from above if (8) is finite, and also bounded from below if $f(x)$ is bounded since

$$
\begin{aligned}
\phi_{0}(x) & \geqq \int_{d}^{x}\left\{\exp \int_{d}^{y}\left[f^{\prime \prime}(t) / f^{\prime}(t)\right] d t\right\} d y \\
& =[f(x)-f(d)] / f^{\prime}(d)
\end{aligned}
$$

by (4) and (5).

Suppose now that (8) diverges, so that $\phi_{0}(x)$ is unbounded from above, and let $\phi_{1}(x)$ be any function which has the properties (3) and (7). By taking a suitable linear combination $\phi(x)=c_{1} \phi_{1}(x)+c_{2}$ we can achieve that $\phi(d)=0, \phi^{\prime}(d)=1$. Now from (3) and (6),

$$
\frac{d}{d x} \log \phi^{\prime}(x) \geqq f^{\prime \prime}(x) / f^{\prime}(x) \geqq \frac{d}{d x} \log \phi_{0}^{\prime}(x)
$$

which implies

$$
\phi^{\prime}(x) \geqq \phi_{0}^{\prime}(x), \quad \phi(x) \geqq \phi_{0}(x) \quad \text { for } x>d .
$$

This shows that $\phi_{0}(x)$ is in a sense the "least convex" among all possible solutions and that $\phi(x)$, hence also $\phi_{1}(x)$, is unbounded.

${ }^{1}$ I am indebted to G. Lorentz for a substantial shortening of the original argument. His proof, which is reproduced above, contributed greatly to a simplified treatment of another part of the paper. 
Theorems 1 and 2 have obvious dual formulations.

THEOREM 1.* Under the same conditions as in Theorem 1, $f(x)$ can be represented in the form

$$
f(x)=\phi_{1}(\psi(x))
$$

where $\phi_{1}$ is convex and $\psi$ concave.

TheoRem 2*. If $f(x)$ is as in Theorem 2 , then it can be represented in the form $\left(1^{*}\right)$ with bounded $\psi(x)$ if and only if

$$
\int_{a}^{d} e^{q(y)} d y
$$

is finite, where

$$
q(y)=\int_{y}^{d}\left[f_{-}^{\prime \prime}(t) / f^{\prime}(t)\right] d t
$$

Here $f_{-}^{\prime \prime}(t)$ denotes $-f^{\prime \prime}(t)$ if $f^{\prime \prime}(t) \leqq 0$ and 0 if $f^{\prime \prime}(t)>0$.

The following example shows that boundedness of $f(x)$ does not necessarily imply finiteness of $(8)$ or $\left(8^{*}\right)$. Take $f(x)=2 x+x^{2} \sin (1 / x)$ over the interval $(0,1)$. It is easily seen that $f^{\prime \prime}(x)$ has a zero $x_{n}$ between $2 /(2 n+1) \pi$ and $2 /(2 n-1) \pi$, and

$$
f^{\prime \prime}(x)\left\{\begin{array}{rrr}
>0 & \text { for } & x_{2 m}<x<x_{2 m-1}, \\
<0 & \text { for } & x_{2 m+1}<x<x_{2 m},
\end{array} \quad m=1,2, \cdots .\right.
$$

It can also be shown easily that

$$
\begin{aligned}
x_{n} & =1 / n \pi+2 / n^{3} \pi^{3}+O\left(n^{-5}\right), \\
f^{\prime}\left(x_{n}\right) & =2-(-1)^{n}+O\left(n^{-2}\right),
\end{aligned}
$$

so that

$$
\int_{x_{2 m+1}}^{x_{2 m}}\left[f^{\prime \prime}(t) / f^{\prime}(t)\right] d t=-\log 3+O\left(m^{-2}\right)
$$

and

$$
q(y)=m \log 3+O(1) \text { for } x_{2 m+1}<x<x_{2 m-1} .
$$

This shows that $\int_{0}^{x_{1}} e^{q(y)} d y$ is divergent.

UNIVERSITY OF ADELAIDE 